\title{
Correlation of $T$ cell response and bacterial clearance in human volunteers challenged with Helicobacter pylori revealed by randomised controlled vaccination with Ty21a-based Salmonella vaccines
}

\author{
T Aebischer, ${ }^{1}$ D Bumann, ${ }^{1}$ H J Epple, ${ }^{2}$ W Metzger, ${ }^{1}$ T Schneider, ${ }^{2}$ G Cherepnev, ${ }^{1}$ \\ A K Walduck, ${ }^{1}$ D Kunkel, ${ }^{2}$ V Moos, ${ }^{2}$ C Loddenkemper, ${ }^{3}$ I Jiadze, ${ }^{2}$ M Panasyuk, ${ }^{4}$ \\ M Stolte, ${ }^{5}$ D Y Graham, ${ }^{6}$ M Zeitz, ${ }^{2}$ T F Meyer ${ }^{1}$
}

- Additional methods, figures and tables are published online only at http://gut.bmj.com/ content/vol57/issue8

${ }^{1}$ Department of Molecular Biology, Max Planck Institute for Infection Biology, Berlin, Germany; ${ }^{2}$ Medical Clinic I, Charite Campus Benjamin Franklin, Berlin, Germany; ${ }^{3}$ Institute for Pathology, Charite Campus Benjamin Franklin, Berlin, Germany; ${ }^{4}$ Kazan State University, Kazan, Russia; ${ }^{5}$ Institute for Pathology, Klinikum Bayreuth, Bayreuth, Germany; ${ }^{6}$ Veterans Affairs Medical Center Houston, Texas, USA

Correspondence to: Professor T F Meyer, Department of Molecular Biology, Max-Planck Institute for Infection Biology, Charitéplatz 1, D-10117 Berlin, Germany; Meyer@mpiib-berlin.mpg.de

TA, DB, HJE, WM and TS contributed equally to this work.

Revised 27 February 2008 Accepted 18 March 2008 Published Online First 16 April 2008

\section{ABSTRACT}

Background: Helicobacter pylori remains a global health hazard, and vaccination would be ideal for its control. Natural infection appears not to induce protective immunity. Thus, the feasibility of a vaccine for humans is doubtful.

Methods: In two prospective, randomised, double-blind, controlled studies (Paul Ehrlich Institute application nos 0802/02 and 1097/01), live vaccines against $H$ pylori were tested in human volunteers seronegative for, and without evidence of, active $H$ pylori infection. Volunteers $(n=58)$ were immunised orally with Salmonella enterica serovar Typhi Ty21a expressing $H$ pylori urease or HP0231, or solely with Ty21a, and then challenged with $2 \times 10^{5} \mathrm{cagPAl}^{-} \mathrm{H}$ pylori. Adverse events, infection, humoral, cellular and mucosal immune response were monitored. Gastric biopsies were taken before and after vaccination, and postchallenge. Infection was terminated with antibiotics.

Results: Vaccines were well tolerated. Challenge infection induced transient, mild to moderate dyspeptic symptoms, and histological and transcriptional changes in the mucosa known from chronic infection. Vaccines did not show satisfactory protection. However, 13 of 58 volunteers, 8 vaccinees and 5 controls, became breath test negative and either cleared $H$ pylori $(5 / 13)$ completely or reduced the $\mathrm{H}$ pylori burden (8/13). $\mathrm{H}$ pylori-specific $\mathrm{T}$ helper cells were detected in 9 of these $13(69 \%)$, but only in 6 of $45(13 \%)$ breath test-positive volunteers ( $p=0.0002$; Fisher exact test). T cells were either vaccine induced or pre-existing, depending on the volunteer.

Conclusion: Challenge infection offers a controlled model for vaccine testing. Importantly, it revealed evidence for $\mathrm{T}$ cell-mediated immunity against $H$ pylori infection in humans.

Half of the world's population is infected with Helicobacter pylori. Infection causes gastritis, peptic ulcer and gastric cancer. ${ }^{1}$ Although infection may be beneficial in some cases, ${ }^{2}{ }^{3}$ its pathological consequences outweigh currently projected beneficial roles. Antibiotic resistance and compliance problems significantly reduce treatment efficacy. ${ }^{4}$ In developing countries, re-infection is common, and current treatment options are inadequate for control. An effective vaccine, however, could prevent re-infection and offer cost-effective infection management.
To date, immunity against $H$ pylori has only been obtained in animal models ${ }^{5}{ }^{6}$ where protection depends, at least in part, on induction of $\mathrm{T}$ helper cells. $^{7}$ In contrast, natural infection in humans appears specifically to inhibit $\mathrm{T}$ cell responses via induction of regulatory $\mathrm{T}$ cells ${ }^{89}$ and direct inhibition of $\mathrm{T}$ cell activation. ${ }^{10-12}$ Although various vaccines have been tested in clinical trials (for reviews see Ruggiero et al. ${ }^{13}$ and Aebischer et $\left.a l^{14}\right)$, it remains unclear whether immunity against $H$ pylori exists in humans and whether vaccination is feasible.

We tested live vaccines based on recombinant Salmonella Ty21a, the licensed typhoid fever vaccine, in volunteers subsequently challenged with $H$ pylori. ${ }^{15}$ Although the vaccines were not satisfactory, the studies revealed clearly that $\mathrm{T}$ cell reactivity against $H$ pylori antigens correlated with clearance or significant reduction of $H$ pylori burden.

\section{METHODS}

Vaccine strain construction and challenge $\boldsymbol{H}$ pylori strain

Construction of recombinant Ty21a vaccines expressing $H$ pylori urease $\mathrm{A}$ and $\mathrm{B}$ subunits (Ty21a(pUreA/B)) or HP0231 (Ty21a(pHP0231), respectively, is described in the supplementary material and in Bumann et al. ${ }^{16}$ Frozen Salmonella of known $10^{10}$ colony-forming units (cfu) were thawed and resuspended in $30 \mathrm{ml}$ of phosphatebuffered saline (PBS) for oral vaccination.

The $H$ pylori challenge strain has been described. ${ }^{15}$ Volunteers were infected with $2 \times 10^{5}$ freshly grown bacteria, resuspended in $30 \mathrm{ml}$ of instant soup (Maggi) for infection. Cfus were confirmed by plating.

\section{Study design}

Two prospective, randomised, double-blind, controlled studies were designed (for diagrams see supplementary figs 2 and 3 ) as a combination of two consecutive protocols: vaccination and challenge infection. Infection was monitored over predefined periods before the application of antibiotic therapy to terminate infection, independent of vaccine effect. The first study was planned as a pilot trial to assess primarily the safety of the approach. The second study was planned based on the outcome of the first trial to assess protective effects.
This paper is freely available online under the BMJ Journals unlocked scheme, see http:// gut.bmj.com/info/unlocked.dtl 


\section{Participants}

The pilot study was conducted in 2003-4. Twenty subjects were enrolled at the Medical Clinic I, Charité Campus Benjamin Franklin, Berlin. Eligible subjects were male, aged between 20 and 50 years, healthy with normal routine blood and chemical laboratory parameters, negative for $H$ pylori infection by $\left[{ }^{13} \mathrm{C}\right]$ urea breath test (UBT), serology and stool antigen test. Exclusion criteria were abnormal upper gastrointestinal tract endoscopy including analysis of biopsies, history of typhoid fever vaccination, history of disease of the biliary or gastrointestinal tract, recent drug prescriptions, allergies to antibiotics, a diet rich in sour or fermented food, cases of gastric cancer in close relatives and regular contact with children younger than 12 years.

The second study was conducted between December 2004 and April 2006. Of 133 subjects assessed and screened as above, 47 volunteers were enrolled at the Medical Clinic I, Charité, Campus Benjamin Franklin, Berlin.

\section{Ethics}

The study protocols were developed adhering to the Declaration of Helsinki, reviewed and approved by the ethical review board of the Charité, Berlin, and the studies were registered with the responsible German federal authority, the Paul Ehrlich Institute (applications nos 0802/02 and 1097/01).

All volunteers were informed about the study protocol, potential risks and adverse reactions to vaccination and challenge infection before giving written consent.

\section{Interventions}

In the first study, there were three treatment arms for vaccination: volunteers were given three doses of $10^{10}$ bacteria orally on day 0,2 and 4 of the Ty21a control $(n=4)$, or Ty21a(pUreA/B) vaccines containing either expression plasmid pDB2 $(n=8)$ or pDB3 $(n=8)$. On day $5,7,10$ and 28 postvaccination, blood was taken for serology and analysis of responses in peripheral blood monocytic cells (PBMCs). Four weeks after vaccination, gastroscopy with biopsy was repeated. Volunteers were interviewed repeatedly to record untoward events.

Because serious adverse events due to challenge could not be excluded, the code was broken in week 6 after vaccination to select one volunteer per treatment arm (volunteer nos 2, 8 and 16) based on a similar response to the Ty21a carrier. Volunteers remained blinded and were challenged with $H$ pylori on day 42 postvaccination and again alerted to report adverse reactions. Infection was monitored by UBT on day 3, 7, 14 and 38 postchallenge and weekly thereafter. Serum was obtained on day 7 and 14, and PBMCs were obtained on day 38 postchallenge. Endoscopy was repeated in week 6 after challenge, and biopsies were taken for $H$ pylori culture, histology, determination of local cytokine secretion and mRNA preparation. Thereafter, $H$ pylori was eradicated by triple therapy for 7 days combining amoxicillin, clarithromycin and pantoprazol (ACP; ZacPac). Eradication was confirmed by UBT on days 68 and 132 after challenge. As gastritis was similar in all three subjects and no severe untoward events were noted, the remaining 17 volunteers were contacted. One could not be contacted and six volunteers declined. Ten volunteers were enrolled for challenge infection which took place 5 months after vaccination. Monitoring and sample collection were performed at the same time intervals postchallenge as before. Therapy was initiated in week 8 instead of week 6 postchallenge.
In the second study volunteers were allocated to a first arm for vaccination with control Ty21a $(n=22)$. Volunteers allocated to a second $\operatorname{arm}(\mathrm{n}=12)$ were given the recombinant Ty21a(pUreA/B) holding plasmid pDB2 used in the first study. Volunteers in the third arm $(n=13)$ were given a novel vaccine strain expressing antigen HP0231, Ty21a(pHP0231). Given capacity constraints, the rationale for introducing the latter and omitting one Ty21a(pUreA/B) vaccine used in the first study was as follows: no differences in immunogenicity were detected between Ty21a(pUreA/B) vaccines; hence only one was selected for further testing. Ty21a(pHP0231) was included because corresponding vaccines were equally efficient in protecting mice compared with the gold standards $H$ pylori lysate and $S$ typhimurium (pureA/B) 22 (TA, DB unpublished). Furthermore, the North American schedule of four vaccine doses given every other day was adopted to maximise responder numbers.

Of the 47 vaccinated volunteers, 45 were infected with $H$ pylori on day 42 as described above. Two withdrew from the study. The observation period after challenge infection was extended to 3 months and instead of blood collection and a gastroscopy on day 28 (postvaccination), blood samples were collected and gastroscopy performed after week 6 and 10 postinfection. Infection was terminated by antibiotic therapy as described above, and success was monitored 1 and 3 months later.

\section{Objectives}

The primary objectives were to assess safety, immunogenicity and potential protective effect of prophylactic vaccination with live, recombinant Salmonella Ty21a vaccines in human volunteers experimentally challenged with $H$ pylori. The secondary objective was to test whether protection, if it existed, was correlated with a particular type of immune response.

\section{Outcomes}

The primary end point was protection from a $H$ pylori challenge - that is, negative testing in four diagnostic tests for active $H$ pylori infection. The four tests were UBT, rapid urease test (RUT) on biopsies, H pylori detection by histochemistry and culture from biopsies.

Secondary outcomes were histological changes and $\mathrm{T}$ and $\mathrm{B}$ cell response patterns, assessed by cytokine and antibody production, respectively. A secondary end point for evidence of protection was re-defined for post hoc analyses and consisted of repeatedly negative UBT and negativity in at least one of the three remaining diagnostic tests.

\section{Sample size}

The first study was designed as a pilot study primarily to assess the safety of the vaccination cum challenge infection model. Sample size was determined based on previous studies with the vaccines used, when $33-56 \%$ of volunteers generated a specific $T$ cell response. Thus, groups of 8 volunteers were immunised with the recombinant vaccines and 4 volunteers were given the control Ty21a. It was expected that $30-50 \%$ of volunteers would generate a urease-specific $T$ cell response and, if this were correlated with protection, the trend should be detectable.

A minimal group size of 20 in the vaccine (cumulated vaccine groups) and 20 in the control groups for the second study was determined using the PS program (http://biostat.mc.vanderbilt. edu/twiki/bin/view/Main/PowerSampleSize) based on the outcome of the first study - that is, indications of clearing response 
in 5 of 9 vaccinees (55\%) and assuming a frequency of $10 \%$ of volunteers in the control groups with a clearing response. The hypothesis that no difference between vaccinees and controls existed was to be tested with a power of 0.8 at a significance level of 0.05 by two-sided Fisher exact test using a dichotomous outcome (protected/non-protected).

\section{Randomisation}

Volunteers were assigned a continuous number upon screening that served as a volunteer ID throughout the studies. To generate a random sequence of vaccine allocation, ID numbers of enrolled volunteers were drawn blindly from a hat by a collaborator not involved in screening or enrolment. The allocation key was concealed and not disclosed to personnel involved in administering the vaccines.

On the days of vaccination, volunteers were given vaccines in ascending order according to their ID, being administered vaccine doses labelled with their ID by the person who generated the allocation key.

The study physician and personnel involved in direct contact with volunteers were blinded and ignorant of the allocation sequence of treatment arms.

\section{Analytical procedures}

Histological analyses were performed as described ${ }^{17}$ by a single pathologist (MS) blinded to treatment keys.

Antibody-secreting cells (ASCs) and specific $\mathrm{T}$ cells were monitored. Cryopreserved PBMCs from the same volunteer collected at different times were thawed and analysed simultaneously. ASCs were enumerated by ELISPOT. ${ }^{18} \mathrm{~T}$ cells were analysed by multichromatic cytofluorimetry ${ }^{19} 20$ after in vitro stimulation of PBMCs with a Salmonella lysate (anticarrier response), and with recombinant urease A (first study) or overlapping peptide pools (15mer peptides with 12mer overlaps) representing urease $\mathrm{A} / \mathrm{B}$ or $\mathrm{HP} 0231$ (see supplementary information). Cells cultured in medium or stimulated with Staphylococcal enterotoxin B served as negative and positive controls, respectively. Responses were compared in PBMCs prepared before vaccination, at day 5 postvaccination, and 6 weeks and 3 months after challenge infection, totalling $>1000$ samples.

Local cytokines were determined in biopsy culture supernatants ${ }^{21}$ by cytometric bead array (CBA system, BD; see supplementary information).

Transcriptional profiling was performed analysing total RNA from antrum biopsies on a custom-made microarray (see supplementary information).

\section{Statistical analyses}

Quantitative parameters of post hoc analyses are presented as medians and range when data sets were not all normally distributed, and groups compared by two-sided, non-parametric tests using Graphpad Prism4.

Significant $\mathrm{T}$ cell responses were determined based on a threshold of mean prevaccination level $+3 \mathrm{SD}$ after testing that prevaccination numbers of memory $\mathrm{CD}_{4} 5 \mathrm{RA}^{-}, \alpha 4 \beta 7$ integrin $^{+}$ $\mathrm{CD}^{+}$cells expressing interferon $\gamma$ (IFN $\gamma$ ) (first study) or CD40L and interleukin 2 (IL2) (second study) were normally distributed (identified outliers in study 2 were excluded from analysis). Volunteers were classified into CD4 $\mathrm{T}$ cell responders and nonresponders and, based on UBT results, as $\mathrm{UBT}^{+}$or $\mathrm{UBT}^{-}$. As a null hypothesis, the independence of UBT results and CD4 $\mathrm{T}$ cell responses was evaluated by two-sided Fisher exact test.
Differences in $\mathrm{T}$ cell response quality between $\mathrm{UBT}^{+}$and $\mathrm{UBT}^{-}$groups were analysed using Statistica 6 software by factorial analysis of data on 14 different $\mathrm{T}$ cell subpopulations (eg, $\mathrm{CD}^{+}$or $\mathrm{CD}^{+}, \mathrm{CD} 40 \mathrm{~L}^{+}$or $\mathrm{CD} 40 \mathrm{~L}{ }^{-}, \mathrm{IFN} \gamma^{+}$or $\mathrm{IFN} \gamma^{-}$cells analysed at four time points and restimulated in vitro with different antigens). The first factor captured $>70 \%$ of the variation in either group and was composed of different $\mathrm{T}$ cell subpopulations.

\section{RESULTS}

\section{Adverse effects of vaccination and challenge infection}

Vaccines were well tolerated, with minor adverse effects such as gastrointestinal nausea $(2 / 20)$, mild diarrhoea $(2 / 20)$ and headaches $(5 / 20)$ reported on single days over a 28 day period (see supplementary table 1). Infection with $H$ pylori caused dyspeptic symptoms such as nausea and abdominal pain that started around day 3 after challenge, but were transient and resolved after 2 weeks in the great majority of volunteers (supplementary fig 2). In each treatment group, one volunteer developed transient grade 3 adverse effects after challenge, with nausea, vomiting, strong abdominal pain and diarrhoea. One volunteer developed a small ventricular ulcer that had completely resolved after 2 weeks of proton pump inhibitor therapy. No serious adverse events were observed, and neither vaccination nor challenge interfered significantly with the volunteers' daily activities.

\section{Course of infection in vaccinated and control-treated volunteers} Infection was monitored by four tests: continuously by UBT, and by RUT, silver staining and culture of biopsy material obtained after 6 weeks and in the third month of infection (second study only). Test results were classified as positive or negative to compute an overall $H$ pylori score $(0=$ all tests negative, $4=$ all positive). Volunteers tested negative in all tests at baseline. Protection as a primary end point was defined as a $H$ pylori score of 0 in week 6.

In the first study, which was designed as a pilot trial, 13 of 20 volunteers vaccinated were infected with $H$ pylori (4 controls and 9 vaccinees) and all developed a positive UBT by day 7 after challenge, indicating reliable and successful infection (fig 1A). Five individuals developed repeatedly negative UBT after week 3 postchallenge (fig $1 \mathrm{~B})$. In week 6 , three of nine vaccinees $(33 \%)$ but none of the four control volunteers had a score of 0 (table 1 ), suggesting a protective vaccine effect. Therefore, protection was tested in a second study.

The second study followed the same design principle to evaluate the live vaccines Ty21a(pureA/B) used in the first trial and a novel vaccine, Ty21a(pHP0231) expressing the $H$ pylori antigen HP0231 that was found in a mouse model to be protective. ${ }^{22}$ Disappointingly, there was no significant difference between vaccine and control groups with regard to the primary end point, $H$ pylori infection (table 2 ).

However, 6 weeks after challenge, the $H$ pylori score was 0 in two volunteers, and six others were negative in two of four tests. Similarly to the first study, after initially testing positive, these volunteers repeatedly tested negative in UBT after week 3 (fig 1C,D). Four volunteers with a $H$ pylori score of $<2$ were observed in the control group and four in the recombinant vaccine-treated groups. Of note, clearance after successful infection has not been observed in 18 volunteers in previous studies designed to define the infectious dose of the present challenge strain. ${ }^{15}$ 
Figure 1 Course of $H$ pylori infection in vaccinated volunteers. A breath test was used to monitor the course of infection in experimentally challenged volunteers over time. (A, B) $\left[{ }^{13} \mathrm{C}\right]$ urea breath test (UBT) results of 4 Ty21a control $(\square)$ and 9 Ty21a(pUreA/B) ( $\mathbf{a}$ ) vaccinated volunteers in the first study. $(C, D)$ UBT results of control $(\square)$, Ty21a(pUreA/B) (ש) and Ty21a(pHP0231) (•) vaccinated volunteers in the second study. Values correspond to mean (SD) UBT results of vaccine groups repeatedly tested at the indicated times postchallenge infection. (B) and (D) UBT results over time of individual volunteers in study 1 and 2, respectively, who although initially testing positive after challenge, tested repeatedly negative (threshold UBT values DoB $<1.5$; stippled line) after week 3 . 'Ch.' and arrow denote time of challenge infection, 'Treatment' and arrow mark the start time of antibiotic eradication. DoB, delta over baseline.
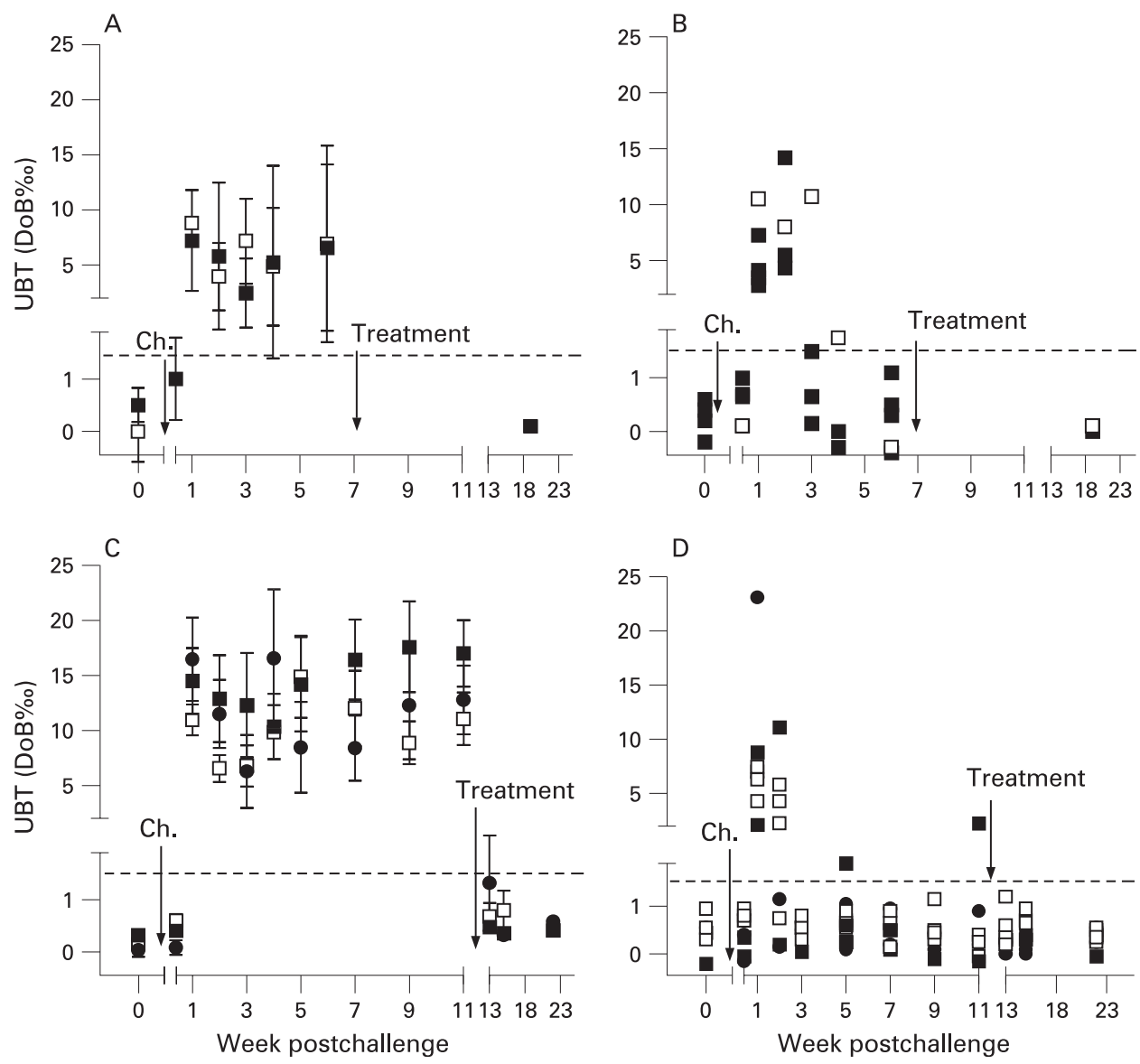

\section{Histological response to vaccination and challenge}

Antrum and corpus mucosa were evaluated for the grade of leucocytic infiltrations, infiltrate activity, grade of regenerative epithelial replacement and mucus depletion. These four parameters were individually scored on a scale of $0-3$ and scores were cumulated to reflect overall histological changes due to vaccination and infection.

Vaccination alone had no detectable effect on the gastric mucosa (cf. supplementary table 2) and did not induce significant changes in local cytokine production (not shown). In contrast, all volunteers developed typical $H$. pylori gastritis $^{15} 6$ weeks after challenge. Importantly, vaccination did not exacerbate inflammation (cf. supplementary tables 2 and 3 for first and second study, respectively). Transcriptional profiling of biopsies taken in week 6 showed that infection increased mucosal chemokine and cytokine mRNA abundance (see supplementary table 4 for a list of all affected mRNAs). In agreement with this, we measured higher levels of IL1- $\beta$, IL2, IL4, IL5, IL6, IL10, IL12, IFN $\gamma$ or tumour necrosis factor $\alpha(\mathrm{TNF} \alpha)$ protein in supernatants of cultured biopsies from that time point (see supplementary fig 5), yet detected no differences in these between treatment groups. Regulatory $\mathrm{T}$ cells expressing the FoxP3 transcription factor ${ }^{23}$ were also enumerated in the mucosa, as these cells are thought to contribute to chronic infection. ${ }^{89}$ FoxP3 $^{+}$cells were rare in uninfected stomach mucosa and the 6-week infected organs, but numbers increased 3 months after infection (supplementary fig 6).

Table 1 Effects of vaccination on $H$ pylori infection (first study)

\begin{tabular}{|c|c|c|c|c|c|c|c|c|c|c|c|c|c|}
\hline \multirow{2}{*}{$\begin{array}{l}\text { Vaccine } \\
\text { Volunteer }\end{array}$} & \multicolumn{4}{|c|}{ Ty21a control } & \multicolumn{9}{|c|}{ Ty21a(pUreA/B) } \\
\hline & 2 & 6 & 7 & 12 & 4 & $8^{*}$ & 10 & $13^{*}$ & 15 & $5^{*}$ & 16 & 18 & 20 \\
\hline \multicolumn{14}{|l|}{ Test $\dagger$} \\
\hline H pylori culture & - & - & + & + & + & - & + & - & + & - & - & - & - \\
\hline RUT & + & - & + & + & + & - & + & - & + & - & - & - & + \\
\hline UBT & + & - & + & + & + & + & + & + & + & - & - & - & - \\
\hline $\begin{array}{l}\text { H pylori } \\
\text { histology }\end{array}$ & + & + & - & + & + & + & + & + & + & - & - & - & - \\
\hline Scoret & 3 & 1 & 3 & 4 & 4 & 2 & 4 & 2 & 4 & 0 & 0 & 0 & 1 \\
\hline
\end{tabular}

*Volunteers receiving the Ty21a(pureA/B) vaccine containing plasmid pDB3.

$\uparrow H$ pylori colonisation was determined by culture of biopsies, RUT on biopsies and Warthin Starry staining of biopsy sections in addition to UBT.

$\$$ Sum of positive tests at 6 weeks and/or 3 months postchallenge; clearing volunteers with a score $\leqslant 2$ are marked in bold. RUT, rapid urease test; UBT, $\left[{ }^{13} \mathrm{C}\right]$ urea breath test. 


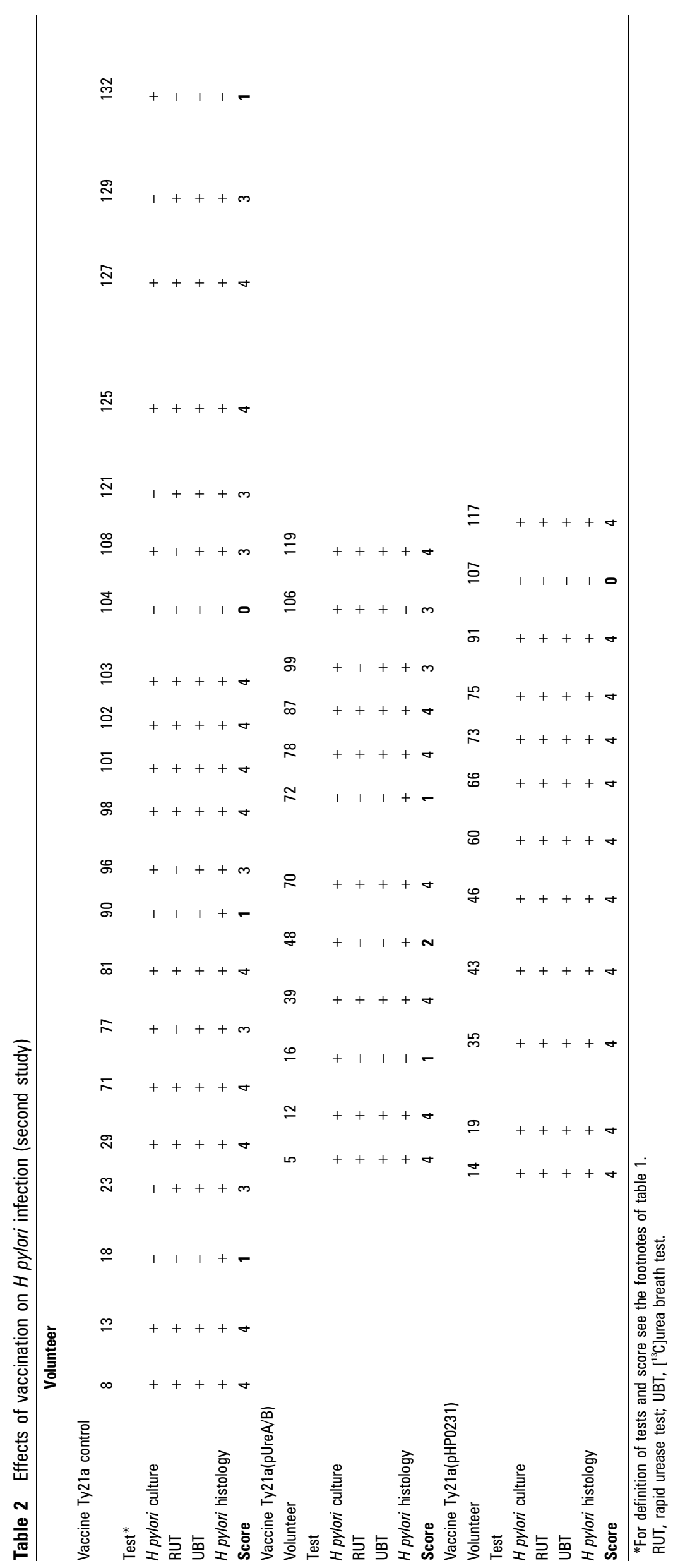

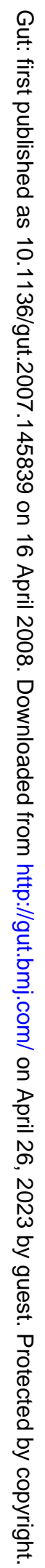




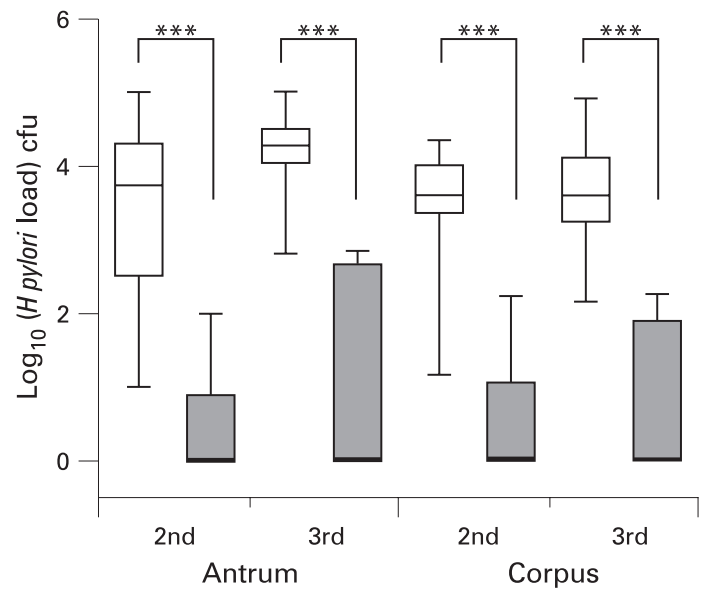

Figure $2 \quad H$ pylori burdens are significantly reduced in $\left[{ }^{13} \mathrm{C}\right]$ urea breath test (UBT)-negative volunteers. Biopsies obtained 6 weeks (second gastroscopy; 2nd) and 3 months (third gastroscopy; 3rd) after infection from the antrum and corpus were processed to determine $H$ pylori colony-forming units (cfu). Data are represented by box and whisker plots which indicate median, range and 25th-75th centiles of the $\log _{10}$ transform of cfu counted per biopsy of the UBT ${ }^{-}$(shaded box) and $\mathrm{UBT}^{+}$ (open box) group of volunteers. Differences in colonisation were highly significant $(p<0.0001$ in all cases; two sided Mann-Whitney test).

\section{Immunological responses}

Salmonella vaccination can induce broad, mucosa-targeted B and $\mathrm{T}$ cell responses. ${ }^{24}{ }^{25} \mathrm{~B}$ cell responses were analysed at day 7 after the first vaccination when ASC numbers against the carrier peak in circulation. Vaccination induced significant numbers of anti-Salmonella-specific ASCs in $25 \%$ of volunteers in the first study and, depending on the group, in $45-75 \%$ in the second study (supplementary table 5). B cell responses to the carrier were in the range expected for these Ty21a vaccines but, as reported, ${ }^{16}{ }^{18}$ vaccine antigen-specific ASCs were below detection.

In contrast, not only Salmonella-carrier but also vaccine antigen-specific - that is, cytokine-producing, eg IFN $\gamma^{+} \mathrm{T}$ cells were detectable by multicolour flow cytometry in in vitro stimulated PBMCs (supplementary table 6). After vaccination (day 5), carrier-specific CD4 ${ }^{+} \mathrm{T}$ cells were detected in $20-25 \%$ of volunteers in both studies. Urease-specific $\mathrm{CD}^{+} \mathrm{T}$ cells were observed in $25 \%$ of Ty $21 \mathrm{a}$ (pureA/B) in study 1 and in $8 \%$ of the respective vaccinees in study 2 . HP0231-specific $\mathrm{T}$ cells were detectable in $33 \%$ of Ty21a(pHP0231)-vaccinated individuals. Antigen-specific $\mathrm{CD}^{+} \mathrm{T}$ cells were not observed (not shown).

After challenge, we recorded urease-specific $\mathrm{T}$ cells in $56 \%$ of Ty21a(pureA/B) vaccinees (up from 25\%) in the first study and $25 \%$ (up from $8 \%$ ) in the second study. In the Ty21a(pHP0231)treated group, an increase was only observed 3 months after infection (to $42 \%$ from $33 \%$ ).

Unexpectedly, $\mathrm{T}$ cells reactive to carrier antigens were detected in five, and against vaccine antigens in six volunteers already before vaccination. In four of the latter, these reactions were against both vaccine antigens, possibly indicating previous exposure to $H$ pylori.

\section{Post hoc analyses}

Comparative analysis of secondary outcomes in $\mathrm{UBT}^{+}$and $\mathrm{UBT}^{-}$ volunteers

While 43 of $58(74 \%)$ volunteers in the two studies remained UBT positive until infections were eradicated, 13 (22\%) tested repeatedly negative and were negative in at least one additional

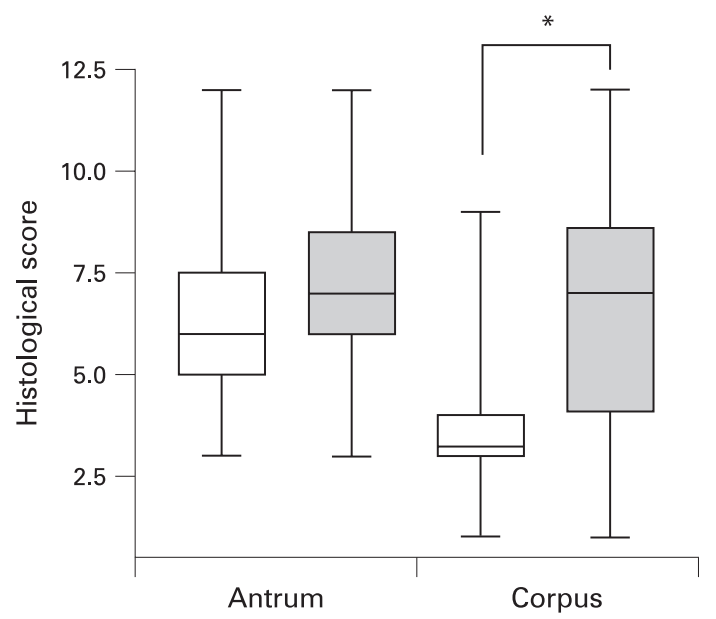

Figure 3 Development of antrum-predominant gastritis in $\left[{ }^{13} \mathrm{C}\right]$ urea breath test (UBT)-positive volunteers 3 months after challenge infection. Gastritis grade, activity, mucus depletion and regenerative changes were scored on a scale of $0-3$. Data are represented in box and whisker plots which indicate median, range and 25th-75th centiles of the cumulative histological scores observed in UBT ${ }^{-}$(shaded box) and UBT' (open box) volunteers (cf. supplememtary tables 3 and 4). Histological scores of corpus gastritis were significantly different between the groups $\left({ }^{*} \mathrm{p}<0.05\right.$; two-sided Mann-Whitney test).

diagnostic test. As this may have indicated active reduction of $H$ pylori burden, a negative UBT and a $H$ pylori score of $<2$ was used to classify volunteers into $\mathrm{UBT}^{-}$and $\mathrm{UBT}^{+}$groups for a post hoc analysis.

We first tested whether $H$ pylori burdens were significantly lower in the so-defined $\mathrm{UBT}^{-}$volunteer group. Indeed $H$ pylori was reduced to $\sim 100 \mathrm{cfu} /$ biopsy or undetectable, and thus was significantly different from the $\sim 10^{4} \mathrm{cfu} /$ biopsy found in $\mathrm{UBT}^{+}$ volunteers (fig 2; $<<0.0001 ;$ Mann-Whitney U test).

We next compared histological parameters (supplementray tables 2 and 3 ) between UBT $^{-}$and $\mathrm{UBT}^{+}$volunteers. Six weeks postchallenge, inflammation of the antrum and corpus showed no significant difference in extent or localisation between these groups. However, 3 months postchallenge, $\mathrm{UBT}^{+}$volunteers had established an antrum-predominant inflammation pattern, a change not observed in the other volunteers (fig 3 ).

In animal models, reduction of bacterial burden requires specific $\mathrm{T}$ cells, and histological correlates such as altered inflammation have been described. ${ }^{26}$ As vaccine antigen-specific, circulating $\mathrm{T}$ cells were determined here (supplementary table $6)$, we tested whether these were correlated with reduced $H$ pylori scores. Four of five volunteers ( $80 \%)$ in the first study and five of eight volunteers $(62 \%)$ in the second study in the UBT ${ }^{-}$ group showed significant $H$ pylori-specific $\mathrm{T}$ cell responses 6 weeks postinfection (fig 4). In contrast, $H$ pylori-specific $T$ cells in PBMCs were detectable in only two of eight (25\%; first study) and four of 37 (11\%; second study) UBT $^{+}$volunteers. The negative correlation between $T$ cell response and UBT positivity was significant in the second study, and was highly significant in the cumulated setting (Fisher exact test: first study $p=0.103$, second study $\mathrm{p}=0.004$; if cumulated, $\mathrm{p}=0.0002$ ).

\section{DISCUSSION}

Despite promise in preclinical models, the feasibility of vaccination against $H$ pylori in humans remains unclear. ${ }^{5}$ In other cases of human-specific pathogens, experimental infection of volunteers for the development of vaccines has provided 
First study

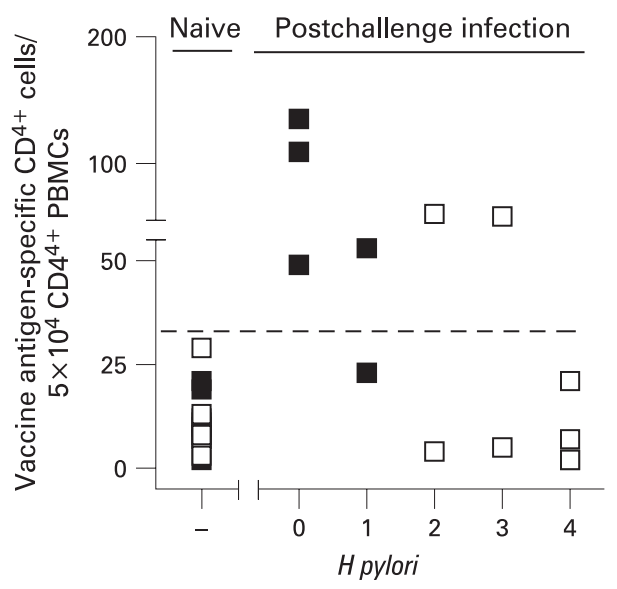

Second study

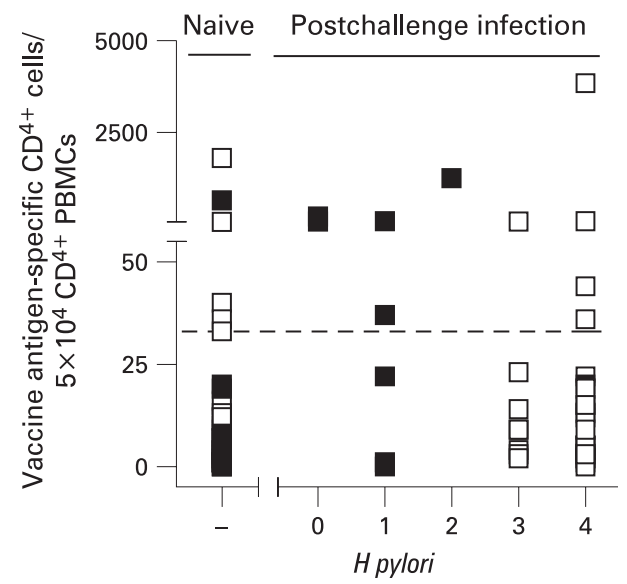

Figure $4 \mathrm{H}$ pylori-specific $\mathrm{CD}^{+} \mathrm{T}$ cell response is negatively correlated with $\mathrm{H}$ pylori infection. Numbers of antigen-specific CD4 ${ }^{+} \mathrm{T}$ cells per $5 \times 10^{4}$ $\mathrm{CD}^{+}$cells detected after short-term in vitro stimulation of $10^{6}$ peripheral blood monocytic cells (PBMCs) obtained before (denoted by naive) and 6 weeks after challenge infection (see supplementary table 6 for exact values) of urea breath test (UBT) ${ }^{-}(\mathbf{\square})$ and UBT ${ }^{+}(\square)$ volunteers were plotted against the overall $H$ pylori score (tables 1 and 2). Stippled lines indicate thresholds (mean of prevaccination values $+3 S D=33$ and 32 in the first and second study, respectively) for significant numbers in specific - that is, cytokine-producing, $\mathrm{CD}^{+} \mathrm{T}$ cells. In the left-hand panel (first study), $y$-values represent interferon $\gamma(\mathrm{IFN} \gamma)^{+}, \beta 7^{+}, \mathrm{CD}^{+} \mathrm{T}$ cell numbers responding against $H$ pylori urease protein. In the right-hand panel (second study), $y$-values indicate $\mathrm{B7}^{+}, \mathrm{CD} \mathrm{OL}^{+}$, interleukin2 (IL2) ${ }^{+} \mathrm{CD}^{+} \mathrm{T}$ cell numbers responding to $\mathrm{H}$ pylori urease and/or HPO231 representing peptide pools. Fisher exact test $p$ values that $\mathrm{CD}^{+} \mathrm{T}$ cell responder proportions were not similar in $\mathrm{UBT}^{-}$and $\mathrm{UBT}^{+}$groups were: first study $\mathrm{p}=0.103$, second study $p=0.004$, cumulated, $\mathrm{p}<0.001$ ).

crucial insight..$^{27-33}$ Here, we chose this approach to clarify the feasibility of vaccinating humans against $H$ pylori. Like others, ${ }^{34}$ we critically evaluated this approach, and alternatives, such as therapeutic vaccination or field trials, were considered but would have had major drawbacks. For example, boosting nonprotective, even pathogenic responses ${ }^{35}{ }^{36}$ in patients who may harbour treatment-resistant $H$ pylori could not be excluded. The use of a defined challenge strain appeared to be advantageous in view of the extreme genetic variance of $H$ pylori in patients.

Safety was of cardinal concern, and three vaccinated volunteers were challenged first to monitor clinical symptoms and tissue response. These were no different from those previously reported for non-vaccinated volunteers. ${ }^{15}$ Histological changes and the transcriptional profile recapitulated essential features of $H$ pylori infection as known from patients, ${ }^{17}{ }^{37}$ and immediate resolution of inflammation even in clearing volunteers was not expected since gastritis also improves relatively slowly after antibiotic eradication. ${ }^{15} 38$ With the caveat that our study protocols only allowed 3 months of observation, we conclude that experimental infection with $H$ pylori enables vaccine testing in a controlled way.

Clearly, the Ty21a-derived vaccines were not satisfactory. Ty21a was used because it is a currently available, licensed human live vaccine and, although strongly attenuated, ${ }^{39}$ it is known to trigger mucosa-homing $\mathrm{T}$ and $\mathrm{B}$ cells. ${ }^{24}{ }^{25}{ }^{40}$ As a carrier, it induced but also boosted vaccine antigen-specific $T$ cells expressing mucosa homing receptors, but not in all volunteers (this study, Bumann et $a l^{16}$ and Metzger et $a l^{18}$ ) which was suboptimal. Novel, more potent $S$ typhi carriers show promise in overcoming this limitation. ${ }^{41} 42$ The immunisation schedule may also be varied to improve induction of mucosa homing $\mathrm{T}$ cell responses. We noted a negative correlation between B cell (ASCs) and $\mathrm{T}$ cell responses against the carrier $\left(r_{\mathrm{sp}}=-0.4705 ; \mathrm{p}=0.0153\right)$. Official Ty21a vaccination schedules were adopted here, but these may favour B cell responses as protection against typhoid fever is positively correlated with the humoral response. Moreover, prime-boost regimens combining live and other vaccine formulations may be more potent, and therapeutic vaccination will also be worth exploring given the boosting effect noted here.

Importantly, we observed significant reduction of $H$ pylori burden which was correlated with $H$ pylori-specific $\mathrm{T}$ cell responses. This could have simply reflected passive loss of infection- "spontaneous clearance"-but in this case detecting correlations with two parameters (inflammation pattern and $\mathrm{T}$ cell response in peripheral blood) would not be expected. However, at present, the possibility that vaccination per se increased local innate and/or adaptive cross-reactive immune defence mechanisms leading to reduced $H$ pylori burden cannot be excluded. In some volunteers, the origin of the $T$ cells was unclear since they had no sign of active or serological evidence of past infection. In others, $\mathrm{T}$ cells were induced by vaccination.

$H$ pylori-reactive $\mathrm{T}$ cells were not exlusively detected in $\mathrm{UBT}^{-}$ volunteers, and it will be important to define markers of a protective response. In animal studies, despite numerous attempts, ${ }^{14}$ markers remain elusive. Results of transcriptome analyses in vaccinated mice have suggested unexpected mediators of immunity, adipokines, ${ }^{43}{ }^{44}$ but mechanisms are not understood. Interestingly, factorial analysis of the multidimensional cytometry data (not shown) from our $\mathrm{T}$ cell analysis suggests that qualitative differences in $\mathrm{T}$ cell responses detectable in PBMCs may exist between volunteers in $\mathrm{UBT}^{+}$and $\mathrm{UBT}^{-}$groups. For example, $\mathrm{CD}_{40 L^{+}}$, IL4 ${ }^{+} \mathrm{CD}^{+} \mathrm{T}$ cells were predominantly associated with the $\mathrm{T}$ cell response in $\mathrm{UBT}^{+}$volunteers. Thus, defining predictive markers of protection may become possible.

Regulatory $\mathrm{T}$ cells may not be a hurdle for prophylactic vaccines as they were recruited into the inflamed stomach mucosa only at later time points, but may close a window of opportunity for therapeutic vaccination. Inversion of the challenge infection and vaccination protocol could help to address this important question in humans.

In summary, this is the first report to show that clearance of $H$ pylori is correlated with an antigen-specific $\mathrm{CD}^{+} \mathrm{T}$ cell response, suggesting immunity against this infection in humans. 
Acknowledgements: We would like to thank S Diescher, M Drabkina, N Gehrmann, K Hoffmann, R Hurwitz, M Kronawitter, S Jackisch, H Mollenkopf, K P Pleissner, $M$ Sörensen and A Zergiebel for expert assistance and technical support, and 0 Liesenfeld for support in processing of stool samples. This work was supported by grants from the Deutsche Forschungsgemeinschaft, KFO 104 Projects 8 (Bu 971/2-1,2, Ae16/4-4 and Z).

\section{Competing interests: None.}

Ethics approval: The study protocols were developed adhering to the Declaration of Helsinki, reviewed and approved by the ethical review board of the Charité, Berlin, and the studies were registered with the responsible German federal authority, the Paul Ehrlich Institute (applications nos 0802/02 and 1097/01).

Patient consent: All volunteers were informed about the study protocol, potential risks and adverse reactions to vaccination and challenge infection before giving written consent.

\section{REFERENCES}

1. Ernst PB, Gold BD. The disease spectrum of Helicobacter pylori: the immunopathogenesis of gastroduodenal ulcer and gastric cancer. Annu Rev Microbiol 2000;54:615-40.

2. Chen Y, Blaser MJ. Inverse associations of Helicobacter pylori with asthma and allergy. Arch Intern Med 2007;167:821-7.

3. Blaser MJ. An endangered species in the stomach. Sci Am 2005;292:38-45.

4. Nakayama Y, Graham DY. Helicobacter pylori infection: diagnosis and treatment. Expert Rev Anti Infect Ther 2004;2:599-610.

5. Del Giudice G, Michetti P. Inflammation, immunity and vaccines for Helicobacter pylori. Helicobacter 2004;9(Suppl 1):23-8.

6. Del Giudice G, Covacci A, Telford JL, et al. The design of vaccines against Helicobacter pylori and their development. Annu Rev Immunol 2001;19:523-63.

7. Ermak TH, Giannasca PJ, Nichols R, et al. Immunization of mice with urease vaccine affords protection against Helicobacter pylori infection in the absence of antibodies and is mediated by MHC class II-restricted responses. J Exp Med 1998;188:2277-88.

8. Raghavan S, Holmgren J. CD4+CD25+ suppressor T cells regulate pathogen induced inflammation and disease. FEMS Immunol Med Microbiol 2005;44:121-7.

9. Rad R, Brenner L, Bauer $S$, et al. CD25+/Foxp $3^{+} T$ cells regulate gastric inflammation and Helicobacter pylori colonization in vivo. Gastroenterology 2006;131:525-37.

10. Gebert B, Fischer W, Weiss E, et al. Helicobacter pylori vacuolating cytotoxin inhibits T lymphocyte activation. Science 2003;301:1099-102.

11. Boncristiano M, Paccani SR, Barone S, et al. The Helicobacter pylori vacuolating toxin inhibits T cell activation by two independent mechanisms. J Exp Med 2003;198:1887-97.

12. Gerhard $\mathbf{M}$, Schmees $\mathbf{C}$, Voland $\mathrm{P}$, et al. A secreted low-molecular-weight protein from Helicobacter pylori induces cell-cycle arrest of T cells. Gastroenterology 2005; 128:1327-39

13. Ruggiero P, Peppoloni S, Rappuoli R, et al. The quest for a vaccine against Helicobacter pylori: how to move from mouse to man? Microbes Infect 2003;5:749-56.

14. Aebischer T, Schmitt A, Walduck AK, et al. Helicobacter pylori vaccine development: facing the challenge. Int J Med Microbiol 2005;295:343-53.

15. Graham DY, Opekun AR, Osato MS, et al. Challenge model for Helicobacter pylori infection in human volunteers. Gut 2004;53:1235-43.

16. Bumann D, Metzger WG, Mansouri E, et al. Safety and immunogenicity of live recombinant Salmonella enterica serovar Typhi Ty21a expressing urease A and B from Helicobacter pylori in human volunteers. Vaccine 2001;20:845-52.

17. Stolte $\mathbf{M}$, Stadelmann 0 , Bethke B, et al. Relationships between the degree of Helicobacter pylori colonisation and the degree and activity of gastritis, surface epithelial degeneration and mucus secretion. Z Gastroenterol 1995;33:89-93.

18. Metzger WG, Mansouri E, Kronawitter M, et al. Impact of vector-priming on the immunogenicity of a live recombinant Salmonella enterica serovar typhi Ty21a vaccine expressing urease $A$ and $B$ from Helicobacter pylori in human volunteers. Vaccine 2004;22:2273-7.

19. Chattopadhyay PK, Yu J, Roederer M. Live-cell assay to detect antigen-specific CD4+ T-cell responses by CD154 expression. Nat Protoc 2006;1:1-6.
20. Maecker HT, Rinfret A, D'Souza P, et al. Standardization of cytokine flow cytometry assays. BMC Immunol 2005;6:13.

21. Schneider T, Zippel T, Schmidt W, et al. Increased immunoglobulin G production by short term cultured duodenal biopsy samples from HIV infected patients. Gut 1998;42:357-61.

22. Sabarth N, Hurwitz R, Meyer TF, et al. Multiparameter selection of Helicobacter pylori antigens identifies two novel antigens with high protective efficacy. Infect Immun 2002;70:6499-503

23. Hori S, Nomura T, Sakaguchi S. Control of regulatory T cell development by the transcription factor Foxp3. Science 2003;299:1057-61.

24. Kantele A, Zivny J, Hakkinen M, et al. Differential homing commitments of antigenspecific T cells after oral or parenteral immunization in humans. J Immunol 1999;162:5173-7.

25. Kantele A, Kantele JM, Savilahti E, et al. Homing potentials of circulating lymphocytes in humans depend on the site of activation: oral, but not parenteral, typhoid vaccination induces circulating antibody-secreting cells that all bear homing receptors directing them to the gut. J Immunol 1997;158:574-9.

26. Blanchard TG, Drakes ML, Czinn SJ. Helicobacter infection: pathogenesis. Curr Opin Gastroenterol 2004;20:10-5.

27. Hornick RB, DuPont HL, Levine MM, et al. Efficacy of a live oral typhoid vaccine in human volunteers. Dev Biol Stand 1976;33:89-92.

28. Hornick RB, Woodward TE, McCrumb FR, et al. Study of induced typhoid fever in man I. Evaluation of vaccine effectiveness. Trans Assoc Am Physicians 1966;79:361-7.

29. Tacket C0, Binion SB, Bostwick E, et al. Efficacy of bovine milk immunoglobulin concentrate in preventing illness after Shigella flexneri challenge. Am J Trop Med Hyg 1992; 47:276-83.

30. Black RE, Levine MM, Clements ML, et al. Experimental Campylobacter jejuni infection in humans. J Infect Dis 1988;157:472-9.

31. Hoffman SL. Experimental challenge of volunteers with malaria. Ann Intern Med 1997;127:233-5.

32. Wang R, Richie TL, Baraceros MF, et al. Boosting of DNA vaccine-elicited gamma interferon responses in humans by exposure to malaria parasites. Infect Immun 2005; 73:2863-72.

33. Garcia L, Jidy MD, Garcia H, et al. The vaccine candidate Vibrio cholerae 638 is protective against cholera in healthy volunteers. Infect Immun 2005;73:3018-24.

34. Michetti P. Experimental Helicobacter pylori infection in humans: a multifaceted challenge. Gut 2004;53:1220-1.

35. Appelmelk BJ, Negrini R, Moran AP, et al. Molecular mimicry between Helicobacter pylori and the host. Trends Microbiol 1997;5:70-3.

36. D'Elios MM, Appelmelk BJ, Amedei A, et al. Gastric autoimmunity: the role of Helicobacter pylori and molecular mimicry. Trends Mol Med 2004;10:316-23.

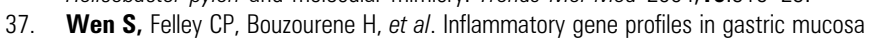
during Helicobacter pylori infection in humans. J Immunol 2004;172:2595-606.

38. Schenk BE, Kuipers EJ, Nelis GF, et al. Effect of Helicobacter pylori eradication on chronic gastritis during omeprazole therapy. Gut 2000;46:615-21.

39. Bumann D, Hueck C, Aebischer T, et al. Recombinant live Salmonella spp. for human vaccination against heterologous pathogens. FEMS Immunol Med Microbiol 2000;27:357-64.

40. Lundin BS, Johansson C, Svennerholm AM. Oral immunization with a Salmonella enterica serovar typhi vaccine induces specific circulating mucosa-homing $\mathrm{CD}^{+}$and CD8 $^{+} \mathrm{T}$ cells in humans. Infect Immun 2002;70:5622-7.

41. Salerno-Goncalves R, Wyant TL, Pasetti MF, et al. Concomitant induction of $\mathrm{CD}^{+}$ and $\mathrm{CD}^{+} \mathrm{T}$ cell responses in volunteers immunized with Salmonella enterica serovar typhi strain CVD 908-htrA. J Immunol 2003;170:2734-41.

42. Wahid R, Salerno-Goncalves R, Tacket CO, et al. Cell-mediated immune responses in humans after immunization with one or two doses of oral live attenuated typhoid vaccine CVD 909. Vaccine 2007;25:1416-25.

43. Mueller A, O'Rourke J, Chu P, et al. Protective immunity against Helicobacter is characterized by a unique transcriptional signature. Proc Natl Acad Sci USA 2003;100:12289-94.

44. Walduck A, Schmitt A, Lucas B, et al. Transcription profiling analysis of the mechanisms of vaccine-induced protection against H. pylori. FASEB J 2004;18:1955-7. 